\title{
Vector mesons in dense matter
}

\author{
Su Houng Lee ${ }^{a b *}$ \\ ${ }^{a}$ GSI, Planckstr. 1, D-64291 Darmstadt, Germany \\ ${ }^{\mathrm{b}}$ Department of Physics, Yonsei University, Seoul 120-749, Korea
}

We will summarize the progress in understanding the changes in the vector meson spectral density in nuclear medium using the constraint equations obtained from the Borel transformed dispersion relation and QCD Operator Product Expansion. We will discuss the results for the scalar mass shift and dispersion effects (three momentum dependence) for the light quark system $(\rho, \omega)$, the strange quark system $(\phi)$ and the heavy quark system $(J / \psi)$ in nuclear medium. For the light quark systems, a nontrivial change in the mass and width are expected, while the dispersion effects are found to be small. Existing model calculations for the dispersion effects are compared to the constraint equation in detail. Very small, but accurate mass shift is obtained for the heavy quark system.

\section{Introduction}

The properties of vector meson in nuclear medium have been the focus of current interest due to their potential role to provide one with a direct observable of the nuclear medium effects, associated with chiral symmetry restoration, via dileptons in $\mathrm{p}-\mathrm{A}$ or A-A reactions [1]. Indeed dileptons from Relativistic Heavy Ion Collisions (RHIC) 2] seemed to suggest a non-trivial change of the vector meson spectral density in a hot/dense environment, which can be understood in terms of model calculations [3] based on decreasing vector meson masses in hot/dense medium[迫, [0. However, some model calculations [6, [7] based on changes of the vector meson spectral densities obtained using effective hadronic model calculations with no decrease in the mass also seem to explain the main features of the CERES data. In all of the approaches, the central question is, how the spectral density changes in hot/dense matter [8]. In this talk, I will discuss the results for the scalar mass shift and dispersion effects (three momentum dependence) of the peak position of the spectral density for the light quark system $(\rho, \omega)$, the strange quark system $(\phi)$ and the heavy quark system $(J / \psi)$ in nuclear medium.

\subsection{Vector mesons in vacuum}

The distinctive features appearing in the dilepton spectrum in $\mathrm{p}-\mathrm{A}$ or $\mathrm{A}-\mathrm{A}$ reactions are the vector meson peaks; the $\rho, \phi$ and the $J / \psi$, whose (mass, width) in the vacuum are $(770,150),(1020,4.4),(3100,0.086) \mathrm{MeV}$. It is interesting to compare the phase spaces of their decay into their corresponding two pseudo particles. For the $\rho$, its two

\footnotetext{
*Alexander von Humboldt Fellow.
} 
pion decay, which accounts for most of the total width, has a large phase space. This is so because the pion is a Goldstone boson and has a small mass. For the $J / \psi$, its decay into D-mesons are forbidden, because the D mesons are not Goldstone bosons and two times its mass is greater than the mass of $J / \psi$. For the $\phi$, the situation is something in between and its decay into two kaons has very little phase space. Hence, chiral symmetry breaking is partly responsible for the large difference in their width. As for the masses of the vector mesons, among other models, QCD sum rules provide an indirect relation to QCD condensates. For the light quark system, $\langle\bar{q} q\rangle$ is dominantly responsible for its mass, for the strange quark system, $\langle\bar{s} s\rangle$ is responsible, and for the $J / \psi$, the charmed quark mass and a small non perturbative contribution from the gluon condensate $\left\langle\frac{\alpha_{s}}{\pi} G^{2}\right\rangle$ are responsible.

Therefore, if chiral symmetry gets restored at finite density or temperature, non-trivial changes will occur to the masses and widths of the vector mesons.

\subsection{Quark condensate at finite density}

The temperature dependence of the quark condensates have been calculated long ago in the lattice [9, 10]. The result is that the light quark condensate will go to zero above the critical temperature. For heavier quark masses, the changes become smaller. At present, due to technical reasons, no lattice result exist at finite density. In all what follows, we will use linear density approximation

$\langle O\rangle_{\rho_{n}}=\langle O\rangle_{0}+\rho_{n} \times\langle O\rangle_{N}$

where $O$ is any operator, $\rho_{n}$ the nucleon density, and the subscripts $\rho_{n}, 0, N$ denotes the nuclear, the vacuum and the nucleon expectation values. Then, we have the following model independent result [11,12] for the quark condensate

$\langle\bar{q} q\rangle_{\rho_{n}}=\langle\bar{q} q\rangle_{0}+\frac{\Sigma_{\pi N}}{2 \hat{m}} \rho_{n} \sim\langle\bar{q} q\rangle_{0} \cdot\left(1-0.2 \frac{\rho_{n}}{\rho_{0}}\right)$,

and for the strange part,

$\langle\bar{s} s\rangle_{\rho_{n}}=\langle\bar{s} s\rangle_{0}+y \frac{\Sigma_{\pi N}}{2 \hat{m}} \rho_{n} \sim\langle\bar{s} s\rangle_{0} \cdot\left(1-0.05 \frac{\rho_{n}}{\rho_{0}}\right)$,

where, $y=2\langle\bar{s} s\rangle_{N} /\left(\langle\bar{u} u\rangle_{N}+\langle\bar{d} d\rangle_{N}\right)$ and $\rho_{0}$ is the nuclear matter saturation density. One notes that already at nuclear matter, we have partial restoration of chiral symmetry; namely, the chiral order parameter is reduced by $20 \%$. Nuclear matter provides a stable environment with non trivial vacuum changes. Hence, if anything happens to the vector mesons at high temperature or density, the tendencies will already be apparent at nuclear matter.

\subsection{Gluon condensate at finite density}

The temperature dependence of the gluon condensates has also been calculated on the lattice 13 16. The result is that it will stay almost constant up to the critical temperature and then reduce to about $60 \%$ of its vacuum value above the critical temperature. The leading density behavior can be obtained from the trace anomaly relation to leading order in $\alpha_{s}$ 匹11]

$T_{\mu}^{\mu}=-\frac{9}{8} \frac{\alpha_{s}}{\pi} G^{2}+\sum m_{q} \bar{q} q$ 
Taking the nucleon expectation value of it and using the most recent determination of the nucleon mass in the chiral limit [17], we have

$$
\left\langle\frac{\alpha_{s}}{\pi} G^{2}\right\rangle_{\rho_{n}}=\left\langle\frac{\alpha_{s}}{\pi} G^{2}\right\rangle_{0} \cdot\left(1-0.05 \frac{\rho_{n}}{\rho_{0}}\right)
$$

Hence, we have a non-trivial change in the gluon condensate, although its relative change is smaller than the case of the quark condensate.

As we have seen, the condensates have non-trivial change in the nuclear medium, which will have a non-trivial effect on the vector meson properties in nuclear medium. Experimentally, there are attempts to produce and observe the decay of vector mesons inside a heavy nuclei and also produce meson bound nuclei. Hence, the theoretical study and the subsequent experimental verification of changes of vector meson properties in nuclear medium will provide a solid basis for understanding the hadronic effects in RHIC and medium effects in general.

\section{QCD constraints}

There have been many model calculations to study vector meson properties in nuclear medium. Here, we will avoid any model calculation and derive a constraint equation that any model calculation should satisfy. The foundation of this approach was laid in ref. [5]

Consider the correlation function of the vector current $J_{\mu}=\bar{q} \gamma_{\mu} q$ at finite density;

$\Pi_{\mu \nu}\left(\omega^{2}, \mathbf{q}^{2}\right)=i \int d^{4} x e^{i q x}\left\langle T\left[J_{\mu}(x) J_{\nu}(0)\right]\right\rangle_{\rho_{n}}$.

Here $q=(\omega, \mathbf{q})$. In what follows, when we give result for explicit vector meson, we will use the currents $J_{\mu}^{\rho, \omega}=\frac{1}{2}\left(\bar{u} \gamma_{\mu} u \mp \bar{d} \gamma_{\mu} d\right)$ for the $\rho, \omega$ mesons, $J_{\mu}^{\phi}=\bar{s} \gamma_{\mu} s$ for the $\phi$ and $J_{\mu}^{J / \psi}=\bar{c} \gamma_{\mu} c$ for the $J / \psi$.

In general, because the vector current is conserved, the polarization tensor in eq.(6) will have only two invariant functions 18 .

$\Pi_{\mu \nu}\left(\omega^{2}, \mathbf{q}^{2}\right)=\Pi_{T} q^{2} \mathrm{P}_{\mu \nu}^{T}+\Pi_{L} q^{2} \mathrm{P}_{\mu \nu}^{L}$,

where we assume the ground state to be at rest, such that, $\mathrm{P}_{00}^{T}=\mathrm{P}_{0 i}^{T}=\mathrm{P}_{i 0}^{T}=0$, $\mathrm{P}_{i j}^{T}=\delta_{i j}-\mathbf{q}_{i} \mathbf{q}_{j} / \mathbf{q}^{2}$ and $\mathrm{P}_{\mu \nu}^{L}=\left(q_{\mu} q_{\nu} / q^{2}-g_{\mu \nu}-\mathrm{P}_{\mu \nu}^{T}\right)$. When $\mathbf{q} \rightarrow 0, \Pi_{L}=\Pi_{T}$, as in the vacuum.

We will make a small $\mathbf{q}$ expansion of the correlation function and look at its energy dispersion relation at fixed $\mathbf{q}$,

$$
\begin{array}{r}
\operatorname{Re} \Pi_{L, T}\left(\omega^{2}, \mathbf{q}^{2}\right)=\operatorname{Re}\left(\Pi^{0}\left(\omega^{2}, 0\right)+\Pi_{L, T}^{1}\left(\omega^{2}, 0\right) \mathbf{q}^{2}+\cdot \cdot\right) \\
=\int_{0}^{\infty} d u^{2}\left(\frac{\rho^{0}\left(u^{2}, 0\right)}{\left(u^{2}-\omega^{2}\right)}+\frac{\rho_{L, T}^{1}\left(u^{2}, 0\right)}{\left(u^{2}-\omega^{2}\right)} \mathbf{q}^{2}+\cdot \cdot\right)
\end{array}
$$

where $\rho\left(u^{2}, \mathbf{q}^{2}\right)=1 / \pi \operatorname{Im} \Pi^{R}\left(u^{2}, \mathbf{q}^{2}\right)$, and $R$ denotes the retarded correlation function. We will construct a constraint equation for $\Pi^{0}, \Pi_{L}^{1}$ and $\Pi_{T}^{1}$. For $\Pi_{L}^{1}, \Pi_{T}^{1}$, we will only look at the "non-trivial" q dependence 19. A simple method to extract the "non-trivial" q dependence is to express the polarization function in terms of $Q^{2}, \mathbf{q}^{2}\left(\Pi\left(Q^{2}, \mathbf{q}^{2}\right)\right)$ and extract the linear $\mathbf{q}^{2}$ term. 
In general for each polarization functions $\left(\Pi^{0}, \Pi_{L}^{1}, \Pi_{T}^{1}\right)$, the OPE [20,21] looks as follows, $\Pi\left(\omega^{2}\right)=\sum_{n} C_{n}\left\langle O_{n}\right\rangle$

Here the $O_{n}$ are operators of (mass) dimension $n$, renormalized at a scale $\mu^{2}$, and $C_{n}$ are the perturbative Wilson coefficients, which for the light quark system can be written as $C_{n}=\frac{c_{n}}{\left(-\omega^{2}\right)^{n / 2}}$ and for heavy quark system as $C_{n}=\frac{c_{n}}{m_{h}^{n}}$.

Let us first discuss the light quark system. After the Borel transformation, the dispersion relation for any one of the polarization function $\left(\Pi^{0}, \Pi_{L}^{1}, \Pi_{T}^{1}\right)$ becomes

B.T. $\operatorname{Re} \Pi\left(M^{2}\right)=\int d s \rho(s) e^{-s / M^{2}}$,

The left hand side, which is the Borel transform (B.T.) of the OPE, looks like the following when including operators up to dimension 6 ,

B.T. $\operatorname{Re} \Pi\left(M^{2}\right)=$ B.T. $\Pi\left(M^{2}\right)_{p e r t}+\frac{c_{4}}{1 ! M^{2}}\left\langle O_{4}\right\rangle+\frac{c_{6}}{2 ! M^{4}}\left\langle O_{6}\right\rangle$

The truncation is valid as long as $M^{2}$ is sufficiently large. The minimum $M_{\min }^{2}$ is usually determined by requiring the correction from higher dimensional operators to be less than $30 \%$ of the perturbative contribution. Now the constraints for the spectral density would be eq.(10), applied above $M^{2}>M_{\min }^{2}$.

As can be seen in eq.(10), the Borel transformation also changes the weighting factor of the spectral density to an $\exp \left(-s / M^{2}\right)$. This has the following advantage for practical applications of our constraint. For small values of the Borel mass, the contribution of the spectral density at larger energy is exponentially suppressed. Consequently, in a model calculation, one can concentrate on the changes of the spectral density near the vector meson mass region and below and model the higher energy part with a simple pole like contribution.

The constraint equation in the vacuum are well satisfied by the spectral density in the vacuum. As we will see, in most cases the changes in the operators $\left\langle O_{n}\right\rangle$ are known. Hence, starting from the vacuum form of the spectral density $(\operatorname{Im} \Pi)$, we can study what changes are consistent with the constraint equation. This provides model independent QCD constraints that any model calculation should satisfy. One can go one step further and try to parameterize the spectral density with a simple delta function type of pole and a continuum and determine the changes in the parameters.

\section{Light quark system $\rho, \omega$}

3.1. $\Pi_{0}$

The operators that dominate the change in the OPE in eq.(10) for the light quark system $(\rho, \omega)$ are the quark operators [5]

$\left\langle O_{4}\right\rangle \rightarrow\left\langle\bar{q} \gamma_{\mu} D_{\nu} q\right\rangle \propto \int d x x[q(x)+\bar{q}(x)]$
$\left\langle O_{6}\right\rangle \rightarrow\left\langle(\bar{q} q)^{2}\right\rangle \propto\left\langle(\bar{q} q)^{2}\right\rangle_{0}+2 \rho_{n}\langle\bar{q} q\rangle_{0}\langle\bar{q} q\rangle_{N}$

The first equation of eq.(12) dominates the changes in the dimension 4 operators and is related to the well known second moment of the quark distribution function. The second 
equation of eq.(12), for which we have used the ground state saturation hypothesis[0], dominates the changes in the dimension 6 operators.

Using a delta function assumption for the spectral density in the constraint equation gives the following result for the scalar mass shift at $\mathbf{q}=0$ [5],

$\frac{m_{V}\left(\rho_{n}\right)}{m_{V}\left(\rho_{n}=0\right)}=1-(0.16 \pm 0.06) \frac{\rho_{n}}{\rho_{0}}$

This result is also consistent with other model calculations 23 25 or the Brown-Rho scaling argument 4 .

A detailed comparison of the constraint equation in eq.(10) with a hadronic calculation, based on chiral SU(3) dynamics with explicit vector mesons were performed in [22]. The result shows a very good agreement between the OPE and the phenomenological spectral density put into the constraint equation in eq.(10). However, the hadronic calculation gave a large increase in width with a small decrease in mass for the $\rho$ and a large decrease in mass with a small increase in width for the $\omega$. Hence only the result for the $\omega$ is consistent with the result in eq.(13). Later it was found that this was a general result, given the uncertainty in the ground state hypothesis for the four quark condensate in the medium 26]; namely, that there exists a band in the mass vs. width plane that satisfies the constraint equation in eq.(10).

\section{2. $\Pi_{T}$}

The constraint for the nontrivial q dependence in eq.(10) has no $\Pi\left(M^{2}\right)_{\text {pert }}$ and has contributions from operators with explicit spin index [19]. The contributions from dimension 4 operators are related to the twist- 2 matrix elements and are well known. The dimension 6 operators are dominated also by the twist- 2 matrix elements. Hence the constraint equation has little uncertainty coming from the OPE. The operators that dominate are

$$
\begin{aligned}
\left\langle O_{4}\right\rangle & \rightarrow\left\langle\bar{q} \gamma_{\mu} D_{\nu} q\right\rangle_{N} \propto \int d x x[q(x)+\bar{q}(x)] \\
\left\langle O_{6}\right\rangle & \rightarrow\left\langle\bar{q} \gamma_{\mu} D_{\nu} D_{\alpha} D_{\beta} q\right\rangle_{N} \propto \int d x x^{3}[q(x)+\bar{q}(x)]
\end{aligned}
$$

Using a delta function assumption for the spectral density and allowing the parameters to change to leading order in density and in $\mathbf{q}^{2}$, we find the following non-trivial momentum dependence in the peak position[19],

$$
\begin{gathered}
\frac{m_{\rho}\left(\rho_{n}\right)}{m_{\rho}\left(\rho_{n}=0\right)}=1-(0.023 \pm 0.007)\left(\frac{\mathbf{q}}{0.5}\right)^{2} \frac{\rho_{n}}{\rho_{0}} \\
\frac{m_{\omega}\left(\rho_{n}\right)}{m_{\omega}\left(\rho_{n}=0\right)}=1-(0.016 \pm 0.005)\left(\frac{\mathbf{q}}{0.5}\right)^{2} \frac{\rho_{n}}{\rho_{0}}
\end{gathered}
$$

where $\mathbf{q}$ is in $\mathrm{GeV} / \mathrm{c}$ unit. This shows a very small momentum dependence compared to the expected scalar mass shift in eq.(13).

A detailed comparison of the constraint equation in eq.(10) for the momentum dependence for the transverse direction has been made 27 to the hadronic calculation, where the vector-meson nucleon scattering amplitude is obtained by resonance saturation in the s-channel. The result shows that the existing model calculations tend to overestimate the 
constraint. This is due to the large $\rho-N-\Delta(1232)$ coupling, which is obtained from the Bonn potential 28]. However, the existing calculations used a non-covariant monopole form factor [6,29] normalized off shell

$F(\mathbf{q})=\frac{\Lambda^{2}}{\Lambda^{2}+\mathbf{q}^{2}}$

On the other hand, the large $\rho-N-\Delta(1232)$ coupling in the Bonn potential is defined with a dipole form factor normalized at the on shell point of the vector meson,

$F_{\rho}\left(q^{2}\right)=\left(\frac{\Lambda_{\rho}^{2}-m_{\rho}^{2}}{\Lambda_{\rho}^{2}-q^{2}}\right)^{2}$.

This reduces the Delta contribution to the rho meson self energy at the invariant mass around $m_{\Delta}-m_{N}$ by approximately a factor of 4 . After this correction, we find that the model calculations give very good agreement with the constraint equation 27.

3.3. $\Pi_{L}$

The constraint for the longitudinal direction is dominated by twist-2 quark and gluon operators $\left\langle\bar{q} \gamma_{\mu_{1}} D_{\mu_{2}} . . D_{\mu_{n}} q\right\rangle_{N} \propto \int d x x^{n-1}[q(x)+\bar{q}(x)],\left\langle G_{\mu_{1}}^{\alpha} D_{\mu_{2}} . . G_{\mu_{n} \alpha}\right\rangle_{N} \propto \int d x x^{n-1} g(x)$,

Using a delta function assumption for the spectral density and allowing the parameters to change to leading order in density and in $\mathbf{q}^{2}$, we find 19 ,

$\frac{m_{V}\left(\rho_{n}\right)}{m_{V}\left(\rho_{n}=0\right)}=1-(0.004 \pm 0.002)\left(\frac{\mathbf{q}}{0.5}\right)^{2} \frac{\rho_{n}}{\rho_{0}}$

for both the $\rho$ and $\omega$. This is a very small effect and no detailed comparison with any hadronic calculations exits yet.

\section{Strange quark system $\phi$}

The OPE in the constraint equation for the $\phi$ meson is dominated by $\left\langle m_{s} \bar{s} s\right\rangle$ for the scalar mass shift and $\left\langle\bar{s} \gamma_{\mu} D_{\nu} s\right\rangle$ for the momentum dependence. Assuming a delta function ansatz for the pole, we find

$\frac{m_{\phi}\left(\rho_{n}\right)}{m_{\phi}\left(\rho_{n}=0\right)}=1-(0.03 \pm 0.015) \frac{\rho_{n}}{\rho_{0}}+(0.0005 \pm 0.0002)\left(\frac{\mathbf{q}}{0.5}\right)^{2} \frac{\rho_{n}}{\rho_{0}}$

for the transverse vector meson and the momentum dependence for the longitudinal $\phi$ is about a factor of two larger.

\section{Heavy quark system $J / \psi$}

For the heavy quark system, we look at the constraints from the moments 30, 31,

$$
\begin{gathered}
\left.M_{n} \equiv \frac{1}{n !}\left(\frac{d}{d \omega^{2}}\right)^{n} \operatorname{Re} \Pi\left(\omega^{2}\right)\right|_{\omega^{2}=-Q_{0}^{2}} \\
=\int_{4 m_{c}^{2}}^{\infty} \frac{\rho(s)}{\left(s+Q_{0}^{2}\right)^{n+1}} d s
\end{gathered}
$$


The changes in OPE in nuclear medium for $M_{n}$ is dominated by the change in the gluon condensate in eq.(5).

To study the $J / \psi$ at rest in the nuclear matter, we will approximate the spectral density with a delta function for the lowest state. This is valid even in nuclear matter, because for a $J / \psi$ at rest, inelastic interactions with nucleons such as $J / \psi+N \rightarrow \bar{D}+\Lambda_{c}$ do not occur. With this assumption, the constraint equation allows for the determination of mass shift of the $J / \psi$ in nuclear matter. We find 32] for the mass shift

$\Delta m_{J / \psi} \simeq-7 \mathrm{MeV}$

This corresponds to small $J / \psi$ - and $\eta_{c}$-nucleon scattering lengths $a=-\mu_{r} \Delta m /\left(2 \pi \rho_{N}\right) \simeq$ $(0.1-0.2) \mathrm{fm}\left(\mu_{r}\right.$ is the meson-nucleon reduced mass). Our results for the mass shifts of the lowest $\bar{c} c$ states are surprisingly close to those reported in ref. 33 36.

Although the expected mass shift is small, the result has little uncertainty coming from OPE and puts reliable constraint on charmonium mass shift which should be met by further studies of heavy quark systems in dense matter.

\section{Conclusion}

We have derived and explored the consequences of model independent constraints for the vector meson polarization at $\mathbf{q}=0$ and $\mathbf{q} \neq 0$ for all the vector mesons $\rho, \omega, \phi, J / \psi$. Most of them have very little uncertainty in the OPE side of the constraint equation and can be used as reliable constraints on all model calculation of the vector meson properties in medium.

\section{Acknowledgments}

I would like to thank B. Friman, T. Hatsuda, H. Kim, S. Kim, F. Klingl, P. Morath and W. Weise for the collaboration, which this talk is based upon. This work was supported in part by KOSEF through grant no. 971-0204-017-2 and 976-0200-002-2 and the Korean Ministry of Education through grant no. 98-015-D00061.

\section{REFERENCES}

1. Quark matter 97, Nucl. Phys. A 638 (1998).

2. G. Agakichiev et al, Phys. Rev. Lett. 75 (1995) 1272; J. P. Wurm for the CERES collaboration, Nucl. Phys. A 590 (1995)103c.

3. G.Q.Li, C.M. Ko and G.E.Brown, Phys. Rev. Lett. 75 (1995) 4007.

4. G.E. Brown and M. Rho, Phys. Rev. Lett. 66 (1991) 2720.

5. T. Hatsuda and Su H. Lee, Phys. Rev. C 46 (1992) R34; T. Hatsuda, Su H. Lee and H. Shiomi, Phys. Rev. C 52 (1995)3364.

6. R.Rapp, G. Chanfray and J. Wambach, Nucl. Phys. A 617 (1997) 472.

7. F. Klingl, W. Weise, hep-ph/9802211.

8. G.E. Brown, G.Q. Li, R.Rapp, M. Rho and J. Wambach, Acta Phys. Polon. B 29 (1998)2309.

9. J. Kogut et.al. , Phys. Rev. Lett. 50 (1983) 393.

10. M. Fukugita and A. Ukawa, Phys. Rev. Lett. 57 (1986) 503. 
11. E.G. Drukarev and E.M. Levin, Prog. Part. Nucl. Phys. 27 (1991) 77.

12. T. D. Cohen, R.J. Furnstahl, D. Phys. Rev. C 45 (1992)1881.

13. M. Campostrini and A. Di Giacomo, Phys. Lett. B 197 (1987) 403.

14. Su H. Lee, Phys. Rev. D 40 (1989) 2484.

15. C. Adami, T. Hatsuda, I. Zahed, Phys. Rev. D 43 (1991)921.

16. V. Koch, G.E. Brown, Nucl. Phys. A 560 (1993)345.

17. B. Borasoy and U.G. Meißner, Phys. Lett. B 365 (1996)285.

18. J. Kapusta, Nucl. Phys. B 148 (1979) 461.

19. Su H. Lee, Phys. Rev. C 57 (1998)927.; Su H. Lee and H. Kim, Nucl. Phys. A 642 (1998) 165.

20. K.G. Wilson, Phys. Rev. 179 (1969) 1499.

21. T. Muta "Foundations of Quantum Chromodynamics", World Scientific, Singapore 1987.

22. F. Klingl, N. Kaiser and W. Weise, Nucl. Phys. A 624 (1997) 527.

23. H.-C. Jean, J. Piekarewicz and A, G, Williams, Phys. Rev. C 49 (1994) 1981; H. Shiomi and T. Hatsuda, Phys. Lett. B 334, (1994) 281.

24. K. Saito and A.W. Thomas, Phys. Rev. C 51 (1995) 2757.

25. B. Friman and M. Soyeur, Nucl. Phys. A 600 (1996) 477.

26. S. Leupold, W. Peters and U. Mosel, Nucl. Phys. A 628 (1998) 311.

27. B. Friman, Su H. Lee and H. Kim, "Constraints on vector mesons with finite momentum in nuclear matter. nucl-th/9903067

28. R. Machleidt, K. Holinde and C. Elster, Phys. Rept. 149 (1987) 1.

29. W. Peters, M.Post, H. Lenske, S. Leupold and U. Mosel, Nucl. Phys. A 632, (1998) 109.

30. M.A. Shifman, A.I. Vainshtein and V.I. Zakharov, Nucl. Phys. B 147 (1979)385; Nucl. Phys. B 147 (1979) 448.

31. L.J. Reinders, H.R. Rubinstein and S. Yazaki, Nucl. Phys. B 186 (1981) 109; Phys. Rep. 127 (1985) 1.

32. F. Klingl, S.Kim, Su H. Lee, P. Morath and W. Weise, nucl-th/9811070.

33. M. Luke, A.V. Manohar, M.J. Savage, Phys. Lett. B 288 (1992) 355.

34. S.J. Brodsky, G.A. Miller, Phys. Lett. B 412 (1997) 125.

35. G.F. de Teramond, R. Espinoza, M. Ortega-Rodriguez, Phys. Rev. D 58 (1998) 034012.

36. A. Hayashigaki, nucl-th/9811092. 